\title{
Formación para la enseñanza del lenguaje oral y escrito en carreras de educación parvularia: variedad de aproximaciones y similares dilemas*
}

\author{
Preparation for teaching oral and written language in early childhood teaching programs: \\ diverse approaches and similar dilemmas
}

\section{Formação de professores para o ensino da linguagem oral e escrita em cursos de educação pré-escolar: variedade de aproximações e dilemas similares}

\author{
Cynthia Adlerstein ${ }^{1}$, Marcela Pardo ${ }^{2}$, Carmen Díaz ${ }^{3}$, Malva Villalón ${ }^{4}$ \\ ${ }^{1}$ Facultad de Educación, Pontificia Universidad Católica de Chile. \\ Teléfono: 56-2-23545391; email: cadlerst@uc.cl \\ ${ }^{2}$ Centro de Investigación Avanzada en Educación, Universidad de Chile. \\ Teléfono: 56-2-29 782593; email: marcelapardo@uchile.cl. \\ ${ }^{3}$ Facultad de Educación, Pontificia Universidad Católica de Chile. \\ Teléfono: 56-2-23545347; email: ddiazo@uc.cl \\ ${ }^{4}$ Facultad de Educación, Pontificia Universidad Católica de Chile. \\ Teléfono: 56-2-23545378; email: malva@uc.cl
}

\begin{abstract}
RESUMEN
Este artículo presenta los resultados de un estudio cuyo objetivo fue analizar la formación para la enseñanza del lenguaje oral y escrito (LOE) en carreras de educación parvularia chilena. Empleando un diseño mixto, para tal propósito se analizaron datos primarios y secundarios referentes al discurso declarado sobre esta área de la formación (planes de estudio, programas de cursos y planteamientos de jefas de carrera). Los resultados obtenidos indican que si bien esta área cobra gran relevancia estructural en las carreras, ello ocurre desde tres perspectivas distintas: disciplinar, crítica y pragmática. Cada aproximación configura variables estructurales y de proceso que le permiten intencionar, desde sus sellos institucionales, las distintas dimensiones del conocimiento práctico para la enseñanza del LOE. Sin embargo, todas las carreras enfrentan restricciones y dilemas similares que se relacionan con la escasez de recursos, las características de los estudiantes y las tensiones propias de nuestro sistema de educación superior.
\end{abstract}

Palabras clave: formación inicial, educadoras de párvulos, conocimiento práctico, lenguaje oral y escrito.

\begin{abstract}
This paper presents the results of a study aimed at analyzing the preparation for teaching oral and written language in Chilean early childhood teaching programs. Based on a mixed method design, the analysis of primary and secondary data focused on the declared discourse within these programs (plans of study, syllabus and head of programs' understandings). The obtained results show that although this area is of great structural significance in teacher education, this occurs from three different perspectives: disciplinary, critical and pragmatic. Each approach
\end{abstract}

Este artículo presenta resultados parciales obtenidos dentro del marco del Proyecto Fondecyt N 1120658 : "Comparación de
la formación inicial para la enseñanza del LOE y conocimiento práctico de las estudiantes de educación parvularia”.

Esta autora agradece también al proyecto FB0003 del Programa de Investigación Asociativa de CONICYT por el financiamiento otorgado. 
frames structural and process variables that develop the dimensions of practical knowledge for teaching oral and written language, according to their own institutional project. However, all programs face similar restrictions and dilemmas that relate to the lack of resources, the characteristics of students and the particular tensions of our higher education system.

Key words: initial preparation, early childhood teachers, practical knowledge, oral and written language.

\section{RESUMO}

Apresentam-se os resultados de um estudo cujo objetivo foi analisar a formação de professores para o ensino da linguagem oral e escrita (LOE) em cursos de educação pré-escolar do Chile. Utilizando-se uma opção metodológica mista, analisaram-se dados primários e secundários referentes ao discurso declarado sobre tal área de formação (planos de estudos, programas de cursos e colocações de coordenadores de curso). Resultados indicam que, ainda que esta área cobre grande importância estrutural nos cursos, ela ocorre desde três perspectivas: disciplinar, crítica e pragmática. Cada aproximação configura variáveis estruturais e processuais que lhes permitem direcionar, desde as características institucionais, as diferentes dimensões do conhecimento prático para o ensino da LOE. No entanto, todos os cursos enfrentam restrições e dilemas similares relacionados à falta de recursos, às características de seus estudantes e às tensões próprias do sistema de educação superior chileno.

Palavras-chave: formação inicial, educadoras de pré-escola, conhecimento prático, linguagem oral e escrita.

\section{INTRODUCCIÓN}

Las educadoras de párvulos cumplen un rol crítico en el desarrollo del lenguaje oral y escrito (en adelante, LOE) de los niños menores de 6 años que asisten a la educación parvularia, al favorecer entre otras habilidades el incremento de su vocabulario y la complejidad de su gramática. Por esta razón, diversos expertos han enfatizado la necesidad de que las educadoras de párvulos reciban una formación inicial sólida para la enseñanza del LOE en la primera infancia (Barnett, 2003; Bredekamp, 2013; Cusumano et al. 2006; Hyson et al. 2013; Neuman, 2013).

Por cierto, abundante y contundente evidencia científica sugiere que el LOE constituye un área de particular relevancia en educación parvularia, al mostrar que los programas que enfatizan el desarrollo del lenguaje oral y escrito se asocian más robustamente con efectos positivos de largo plazo sobre los niños que asisten a ellos, tanto en términos de su desarrollo y aprendizaje actual, como de su posterior desempeño en el sistema escolar (Burchinal et al. 2002; Shonkoff \& Phillips, 2000).

No obstante, según han consignado prominentes especialistas del área (Bredekamp, 2000; Strickland et al. 2002), la formación de educadores (en general, y de las educadoras de párvulos, en particular) tiende a no abordar, ni en términos de extensión ni de profundidad, los contenidos necesarios para la adecuada enseñanza del LOE en la primera infancia.

De manera similar, la literatura no identifica programas ejemplares de formación de educadoras de párvulos en cuanto a la enseñanza del lenguaje oral y escrito, dando cuenta de la falta de un estándar dorado en esta área (Bowman et al. 2000; Whitebook et al. 2012). Como ejemplo de esta situación, las mallas curriculares de doce programas de educación parvularia internacionalmente prestigiosos, muestran una gran heterogeneidad en términos del porcentaje de cursos destinados a la formación en el área del lenguaje oral y escrito, el que fluctúa entre 0\% y 33\% (Bank Street College, 2013; Boston College, 2013; Institute

\footnotetext{
Es necesario señalar que esta revisión se basó en la información sobre la estructura de las mallas curriculares disponible en los respectivos sitios web de los programas revisados, sin disponer de información sobre el número de créditos asignado a
} 
of Education University of London, 2013; Mills College, 2014; Monash University, 2013; National Institute of Education, 2013; Teachers College, 2014; Universidad de Ciencias Pedagógicas Enrique José Varona, 2013; University of Cambridge Faculty of Education, 2014; University of Oulu, 2013; University of Toronto, 2013; Wheelock College, 2013).

De tal manera, si bien existe evidencia abundante sobre los procesos que configuran el desarrollo del LOE en la primera infancia, junto con propuestas sobre los conocimientos con que deben contar las educadoras de párvulos para favorecer dichos procesos, se constata un vacío importante respecto a cómo incorporar dichos conocimientos en la formación de educadores (Fillmore \& Snow, 2000; Neuman, 2013). Este vacío ha sido explícitamente reconocido como más serio para el caso de la formación de educadoras de párvulos, por tratarse de profesionales a cargo de la educación de niños que se encuentran en pleno proceso de adquisición de la lengua (Bredekamp, 2003).

Para el caso de Chile, un muy reducido número de investigaciones se ha referido a la formación de educadoras de párvulos para la enseñanza del lenguaje oral y escrito. Al respecto, los análisis de mallas curriculares de un conjunto de carreras, realizados por García-Huidobro (2006) y por Romo y colegas (Romo et al. 2009), han constatado que la enseñanza del LOE sí está presente, en proporción variable, en la mayor parte de las carreras de formación, aunque no en todos ellos. Similares hallazgos se han realizado para las carreras de pedagogía en enseñanza básica (Manzi, 2011; Sotomayor et al. 2013).

$\mathrm{El}$ presente estudio se propuso contribuir a expandir el conocimiento actual sobre la formación inicial de educadoras de párvulos para la enseñanza del lenguaje oral y escrito, introduciendo en el análisis un parámetro interpretativo que permite superar la sola consideración de variables estructurales (i.e. cuantificación de cursos) que guió los estudios nacionales previos ya citados (Garcia-Huidobro, 2006; Romo et al. 2009; Sotomayor et al. 2013). Concretamente, este estudio buscó describir la formación de educadoras de párvulos para la enseñanza del LOE en función del desarrollo del "conocimiento práctico" (Meijer et al. 2002) (este concepto se discute en la siguiente sección). El conocimiento práctico se refiere al tipo de conocimientos que es peculiar de los educadores y que la investigación de las dos últimas décadas ha subrayado como fundamental en la formación inicial de estos profesionales (Calderhead, 1991; Cochran-Smith, 2010; Grossman, 1990, 2005; Munby et al. 2001; Shulman \& Shulman, 2004; Spodek \& Saracho, 1990).

Al respecto, resulta interesante destacar que escasos estudios sobre el concepto de conocimiento práctico -y sus equivalentes- han sido realizados con educadoras de párvulos (Genishi et al. 2001), tendiendo a concentrarse en docentes de enseñanza básica y media (Burney, 2004; Cochran et al. 1993; Connelly et al. 1997; Fernández-Balboa \& Stiehl, 1995; Howey \& Grossman, 1989; Marks, 1990; Meijer et al. 2002; Shulman, 1987), por lo que el presente estudio representa un aporte también en estos términos, al expandir el uso de este concepto hacia este grupo distintivo de educadores.

cada curso ni sobre las unidades de aprendizaje de cada curso. Por otra parte, se debe notar también que esta revisión incluyó programas de nivel de pregrado y postgrado. 


\section{MARCO TEÓRICO}

\subsection{DEBILIDADES EN LA FORMACIÓN DE EDUCADORAS DE PÁRVULOS PARA LA ENSEÑANZA} DEL LENGUAJE ORAL Y ESCRITO

Varios autores consignan que en su formación inicial, muchos educadores (en general, incluyendo a educadores de educación parvularia, básica y media) no han recibido la preparación para adquirir los conocimientos que consensualmente se consideran relevantes para la enseñanza del LOE. Reconociéndolo como una falencia seria, han planteado la necesidad de que las universidades introduzcan estos elementos en la formación de educadores (Bredekamp, 2000; Feldman, 2003; Neuman, 2013).

Como posible explicación al respecto, se ha planteado que ello puede deberse a la falta de especialistas al interior de muchos programas de formación de educadores, debido a lo cual los cursos que abordan el LOE o no alcanzan la profundidad requerida o no están adaptados debidamente a las necesidades pedagógicas de los futuros educadores (Fillmore $\&$ Snow, 2000).

Otra posible razón es la tensión entre la formación generalista que tradicionalmente se ha impartido en las carreras de formación de educadoras de párvulos y de docentes de enseñanza básica, por un lado, y la formación en profundidad que requieren algunas áreas, como el LOE. Concretamente, dentro del abarcador plan de estudios de estos educadores la formación en el área del LOE compite, en términos de tiempo asignado, con otras materias también relevantes; por lo tanto, si el contenido sobre el LOE se expande en la formación de educadores, otro contenido que actualmente es parte del plan de estudios deberá ser eliminado o reducido (Bredekamp, 2000).

Por esta razón, Feldman (2003) ha propuesto que, para fortalecer la formación de educadores en el área del LOE, no sólo se requiere evidencia científica sólida que avale esta necesidad, sino también consenso político amplio sobre la importancia de asignarle relevancia en los planes de estudio.

En un esfuerzo por delinear el contenido sobre el LOE que debiese formar parte de las carreras de formación de educadores, Fillmore \& Snow (2000) han propuesto un listado de cursos potencialmente relevantes, los cuales en su conjunto, abordan los temas fundamentales sobre la enseñanza del lenguaje oral y escrito. Estos cursos son: Lenguaje y lingüística; Lengua y diversidad cultural; Sociolingüística; Desarrollo del lenguaje; Segunda lengua; Discurso académico; Análisis de textos.

Complementariamente, Valdés et al. (2005) han sugerido que en la formación de los educadores de párvulos se debería incorporar un curso introductorio de lingüística centrado en la enseñanza y el aprendizaje, y una planificación cuidadosa para apoyar a los estudiantes en la comprensión del desarrollo del lenguaje oral, como también, de la lectura y la escritura. Estos autores sugieren, además, que esta disciplina sea dictada por el departamento de lingüística, a cargo de especialistas que conozcan y comprendan los problemas que enfrentan los educadores en las escuelas. Se sugiere incorporar en el curso señalado, el lenguaje en uso en la vida cotidiana, incorporando los bloques de construcción de idioma que se presentan, como el sistema de sonido (fonológico), la estructura de las palabras (morfológico) y la estructura de oraciones (sintáctico). Se sugiere además, que la segunda parte de cada curso podría cubrir el primer y segundo lenguaje de adquisición, la variación del lenguaje y la relación entre lenguaje y alfabetización, como también la relación entre lenguaje oral y escrito. 


\subsection{LO QUE LAS EDUCADORAS DE PÁRVULOS REQUIEREN SABER PARA LA ENSEÑANZA DEL} LENGUAJE ORAL Y ESCRITO

Existe amplio consenso en torno al conocimiento con que los educadores, de distintos niveles de enseñanza, debieran contar para desarrollar competencias en lenguaje y alfabetización de los niños, adolescentes y jóvenes a su cargo (Burns et al. 1999; National Institute of Child Health and Human Development, 2000). No obstante, las referencias específicas a educadoras de párvulos son escasas, incluyéndoselas dentro de tratados más amplios que pueden ser aplicados al caso de la educación parvularia (Bredekamp, 2003; International Reading Association - National Association for the Education of Young Children, 1998).

De acuerdo con dos amplias y exhaustivas síntesis sobre el tema, los educadores debiesen tener un conocimiento amplio y profundo sobre el desarrollo del lenguaje y cómo éste puede ser favorecido en contextos educativos. Remarcando que ello no implica que los educadores deban ser expertos en lingüística, las autoras de estos trabajos explican que se trata más bien de que comprendan el uso del lenguaje en la vida cotidiana y los conceptos que lo explican (Adger et al. 2003; Fillmore \& Snow, 2000).

Los conocimientos sobre el LOE que Fillmore \& Snow (2000) plantean que los educadores debieran asumir, son los que se sintetizan en la Tabla 1 a continuación.

Tabla 1: Conocimientos sobre el Lenguaje Oral y Escrito

\begin{tabular}{|ll|ll|}
\hline \multicolumn{2}{|c|}{ Lenguaje Oral } & \multicolumn{1}{c|}{ Lenguaje Escrito } \\
\hline - & Las unidades básicas del lenguaje & $\bullet$ & Ortografía \\
- & Regularidades del lenguaje & - & Complejidades de la lectura \\
- Adquisición del léxico & Lenguas vernáculas & Dificultades con la escritura narrativa y \\
- & Lenguaje académico & & Cómo evaluar un fragmento escrito \\
- & Segunda lengua & - & Complejidad de un texto \\
\hline
\end{tabular}

Por su parte, Neuman \& Kamil (2010) sostienen que en la formación de los educadores que trabajan en alfabetización temprana, se deben integrar en el currículo las competencias del lenguaje oral, las experiencias de alfabetización temprana, la alfabetización de la familia en el aprendizaje de la lectura, el desarrollo de vocabulario y el conciencia fonológica; como también el manejo en el uso de variados métodos de instrucción de acuerdo a las edades y desarrollo apropiado según las necesidades.

\subsection{EL CONOCIMIENTO PRÁCTICO DE LAS EDUCADORAS DE PÁRVULOS}

La investigación científica de las tres últimas décadas ha mostrado que el conocimiento que poseen los docentes, lejos de ser puramente disciplinar, se caracteriza por ser de naturaleza intuitiva, creativa, práctica y altamente personalizada. De acuerdo con esto, distintos conceptos han intentado sintetizar las peculiaridades del conocimiento docente, como los de "conocimiento artesanal" ("craft knowledge") (Brown \& McIntyre, 1992; 
McNamara \& Desforges, 1978), "conocimiento práctico personal” ("personal practical knowledge") (Clandinin y Connelly, 1988), "conocimiento pedagógico del contenido" ("pedagogical content knowledge") (Shulman, 1987) y "conocimiento práctico" ("practical knowledge") (Elbaz, 1983).

Estas conceptualizaciones buscan avanzar respecto de aquellas que descansan principalmente en la dimensión racionalista del conocimiento, y que, según criterios científicos de validez y confiabilidad, han pretendido codificar el conocimiento de los educadores en leyes generales que permitan alta predictibilidad y control (Freidson, 1986).

El tipo de conocimiento que es propio de los educadores ha sido escasamente estudiado para el caso de las educadoras de párvulos. Para explicar este relativo rezago, Genishi y colegas (Genishi et al. 2001) han postulado tres razones. La primera es que sólo recientemente se ha comenzado a reconocer la necesidad de que las educadoras de párvulos cuenten con una sólida base de conocimientos, equivalente a la que requieren los educadores de enseñanza básica y media. La segunda razón es que la importancia asignada por el campo de la educación parvularia a la psicología del desarrollo ha restado valoración a otros elementos también necesarios para el ejercicio de las educadoras de párvulos. La tercera es que, tradicionalmente, este campo ha restado importancia a los contenidos relevantes para la educación parvularia, por asociarlos con la prematura escolarización de la primera infancia.

Dentro del referido conjunto de conceptos, el de "conocimiento práctico" resulta especialmente apto para estudiar la formación de educadoras de párvulos, ya que se compone de un conjunto de dimensiones que abarcan la diversidad de situaciones que forman parte del trabajo en el aula, en correspondencia con la aproximación holística de la enseñanza, la que constituye un principio fundamental en educación parvularia (Bowman et al. 2000; Saracho \& Spodek, 2001). Así, Meijer et al. (2002) han identificado seis dimensiones que integran el conocimiento práctico:

- Conocimiento de la materia, la cual dice relación con el conocimiento del LOE en lo que refiere específicamente a la teoría, no directamente relacionado con la enseñanza.

- Conocimiento sobre el desarrollo y el aprendizaje de los estudiantes, en términos generales, no directamente relacionado con el LOE.

- Conocimiento sobre las características de los párvulos, relevantes para el aprendizaje del LOE.

- Conocimiento sobre los propósitos, la que alude a la relevancia y los objetivos asociados a la enseñanza del LOE.

- Conocimiento sobre el currículum, la cual se refiere a los textos y materiales usados en las actividades pedagógicas sobre LOE.

- Conocimiento de técnicas instruccionales, la que alude al diseño, preparación y estructura de las actividades pedagógicas para la enseñanza del LOE.

En este marco, el concepto de conocimiento práctico se define como el cuerpo de conocimiento (declarativo y procedimental) y de creencias (incluyendo valores y normas) que orientan el quehacer de los educadores, al proporcionarles una comprensión holística sobre las situaciones de aula y sobre los dilemas que enfrentan para llevar a cabo las experiencias pedagógicas (Krieg, 2010; Lenz Taguchi, 2007; Maakestad Wolf, 1999; McLean, 1992; Mischo et al. 2014). 
El conocimiento práctico lo generan los educadores mismos, como resultado de la integración y mutua influencia de distintas fuentes, emanando así de la teoría y de su propia práctica. Sus características centrales son las siguientes: (a) es personal -el conocimiento práctico de cada educador es singular en alguna medida; (b) es contextual -está definido y adaptado a la situación de aula; (c) está basado en (la reflexión sobre) la experiencia -se origina y se desarrolla en la experiencia de enseñanza; (d) es tácito -los educadores pueden no ser capaces de articular verbalmente su conocimiento práctico; (e) guía la práctica de los educadores, no sólo proviene de ella; (f) se relaciona con el contenido -está conectado con la materia que se enseña (Beijaard et al. 2004; Beijaard \& Verloop, 1996; Beijaard, 1995; Meijer et al. 1999, 2001; Meijer et al. 2002; Ponte et al. 2004; Van Driel et al. 2001).

\section{DISEÑO METODOLÓGICO}

El diseño de este estudio corresponde a una metodología cualitativa de nivel descriptivo. Teniendo como unidad de análisis las carreras de formación de educadoras de párvulos, se buscó identificar las transversalidades y particularidades de cada una, a través de un diseño mixto (Hernández Sampieri et al. 2007, 2010; Tashakkori \& Teddle, 2003).

\subsection{MUESTRA Y CRITERIOS DE SELECCIÓN}

La muestra incluyó 10 carreras de educación parvularia, seleccionadas según un criterio de accesibilidad (Ministerio de Educación, 2014), quedando delimitada la selección de los casos a la Región Metropolitana. Asimismo, de acuerdo con criterios de relevancia, fue necesario seleccionar carreras con un reconocido o aceptado grado de consolidación de su calidad.

Considerando el creciente consenso sobre la falta de confiabilidad de los indicadores existentes para determinar la calidad de las carreras de pedagogía en general (Domínguez et al. 2012; Panel de Expertos para una Educación de Calidad, 2010), se decidió intencionar esta selección de la muestra a través del Método Delphi (Sahakian, 1997).

De acuerdo con ello, once académicos nacionales -especialistas en temas de educación superior y en formación inicial de educadoras de párvulos-fueron consultados en dos rondas sucesivas de entrevistas semi-estructuradas. Esta consulta tuvo como propósito identificar criterios que permitieran valorar la calidad de las carreras de formación de educadoras de párvulos del país -y que debían ser integrados en el muestreo-, ponderando el peso relativo de cada uno. Como resultado de este ejercicio, se obtuvo una muestra conformada por diez carreras de formación de educación parvularia, que mostrando distintos grados de consolidación, cumplían con una calidad considerable dentro de su campo.

\subsection{RECOLECCIÓN DE DATOS}

El estudio recolectó datos primarios y secundarios en torno al discurso declarado de las carreras seleccionadas sobre la formación para la enseñanza del LOE. Los datos primarios estuvieron constituidos por los planteamientos de las respectivas jefas de carrera, recabados mediante entrevistas semi-estructuradas individuales, en torno a los lineamientos estratégicos que configuran la formación en esta área. Los datos secundarios 
estuvieron constituidos por los correspondientes planes de estudio, las mallas curriculares y los programas de los cursos que las jefas de carrera identificaron como relevantes para la formación en el área del lenguaje oral y escrito. Ambas fuentes dan cuenta de un currículum prescrito y de un enfoque formativo declarado que opera a nivel de estructura, procesos y orientaciones (Viernickel y Tietze, 2010), aun cuando ello no necesariamente refleja el desarrollo cotidiano del currículum al interior de las carreras analizados.

Las pautas de entrevista se estructuraron según categorías apriorísticas definidas en torno a las dimensiones de conocimiento práctico descritas por Meijer et al. (2002), reoperacionalizadas para la educación parvularia. Esta redefinición de las categorías se realizó en base al juicio del experto y al tecnolecto del campo profesional nacional, según se describe en la Tabla 2:

Tabla 2: Operacionalización de las categorías del Conocimiento Práctico

\begin{tabular}{|c|c|}
\hline $\begin{array}{l}\text { Dimensión del Conocimiento } \\
\text { Práctico intencionada en la } \\
\text { formación para el LOE }\end{array}$ & Operacionalización \\
\hline $\begin{array}{l}\text { Conocimiento } \\
\text { de la Materia }\end{array}$ & $\begin{array}{l}\text { - Conocimiento delLOEen loque refiere específicamente } \\
\text { a la teoría del lenguaje y la literatura, no directamente } \\
\text { relacionado con su enseñanza. } \\
\text { Ejemplos: Campos del lenguaje, géneros literarios, } \\
\text { teorías de adquisición y desarrollo de la lengua. }\end{array}$ \\
\hline $\begin{array}{l}\text { Conocimiento } \\
\text { del Desarrollo y Aprendizaje }\end{array}$ & $\begin{array}{l}\text { - Conocimiento general sobre el desarrollo y el } \\
\text { aprendizaje en la primera infancia, no directamente } \\
\text { relacionado con el aprendizaje del LOE. } \\
\text { Ejemplos: teorías cognoscitivistas, teorías } \\
\text { socioculturales. }\end{array}$ \\
\hline $\begin{array}{l}\text { Conocimiento } \\
\text { del Párvulo }\end{array}$ & $\begin{array}{l}\text { - Conocimiento de características de los párvulos a su } \\
\text { cargo, relevantes para el desarrollo y el aprendizaje del } \\
\text { LOE en la primera infancia. } \\
\text { Ejemplos: Intereses, conocimientos previos, hábitos, } \\
\text { fortalezas y limitaciones de los niños; pautas de } \\
\text { crianza y expectativas familiares; antecedentes } \\
\text { sociodemográficos de su núcleo familiar. }\end{array}$ \\
\hline $\begin{array}{l}\text { Conocimiento } \\
\text { de los Propósitos }\end{array}$ & $\begin{array}{l}\text { - Importancia y objetivos que se le atribuyen a la } \\
\text { enseñanza del LOE en la primera infancia. } \\
\text { Importancia y objetivos que se le atribuyen a la } \\
\text { formación de educadores de párvulos para la enseñanza } \\
\text { del LOE. } \\
\text { Ejemplos: Comunicación con otros, ciudadanía } \\
\text { infantil, desarrollo del pensamiento, integración de las } \\
\text { familias. }\end{array}$ \\
\hline
\end{tabular}




\begin{tabular}{|c|c|}
\hline $\begin{array}{l}\text { Conocimiento } \\
\text { del Currículum }\end{array}$ & $\begin{array}{l}\text { - Referentes curriculares empleados para el trabajo } \\
\text { pedagógico y/o modalidades curriculares que } \\
\text { estructuran las decisiones de enseñanza del LOE. } \\
\text { Ejemplos:BasesCurricularesdelaEducaciónParvularia, } \\
\text { (en adelante, BCEP), Programas Pedagógicos para } \\
\text { Primer y Segundo Nivel de Transición, Mapas de } \\
\text { Progreso de la Educación Parvularia, modalidades } \\
\text { curriculares para la educación parvularia (Montessori, } \\
\text { High-Scope, Reggio Emilia e Integral), prescripciones } \\
\text { curriculares, currículum oculto. }\end{array}$ \\
\hline $\begin{array}{l}\text { Conocimiento } \\
\text { de Estrategias Pedagógicas }\end{array}$ & $\begin{array}{l}\text { - Técnicas para la planificación, implementación y } \\
\text { evaluación de los contextos de aprendizaje utilizado } \\
\text { para la enseñanza del LOE. } \\
\text { Ejemplos: estrategias de organización del espacio, } \\
\text { como rincón de lectura, sala letrada, biblioteca de aula, } \\
\text { momento de lectura silenciosa sostenida u hora del } \\
\text { cuento. Estrategias de distribución de libros y textos, } \\
\text { como cuento viajero, sistema de préstamo de libros. } \\
\text { Estrategias de producción textual, como escritura a } \\
\text { través del maestro y escritura por sí mismo. }\end{array}$ \\
\hline
\end{tabular}

\subsection{ANÁLISIS DE DATOS}

El análisis de los planes de estudio, mallas curriculares y programas de curso sobre LOE buscó estimar la proporción que representan los cursos sobre LOE, dentro de la trayectoria formativa de cada carrera. Así también, se buscó estimar el número de objetivos/unidades de aprendizaje sobre LOE dentro de los programas de los cursos analizados. Este método de análisis complejiza las contabilizaciones de cursos realizadas en estudios anteriores nacionales (Garcia-Huidobro, 2006; Manzi, 2011; Rojas et al. 2008; Sotomayor et al. 2013), al introducir en el análisis los planes de estudio y los planteamientos de las jefas de carrera, recogiendo variables de orientaciones y proceso (Viernickel y Tietze, 2010) en la línea de los trabajos de Early y Winton (Early \& Winton, 2001), Ray y colegas (Ray et al. 2006), Whitebook y colegas (Whitebook et al. 2012; Whitebook et al. 2005; Whitebook et al. 2009). Además, como una innovación propia del presente estudio, el análisis de los cursos sobre LOE se desarrolló a partir de las categorías apriorísticas basadas en las seis dimensiones del conocimiento práctico (Meijer et al. 2002). Estas categorías también orientaron el análisis de las entrevistas a las jefas de carrera, para lo cual se empleó el Método de Comparación Constante (Strauss y Corbin, 2002), en términos de codificación abierta y axial de los discursos.

\section{RESULTADOS}

Los hallazgos de este estudio pueden sintetizarse en tres grandes ideas, que se desarrollarán en profundidad más adelante: 1) Las carreras analizadas asignan gran 
relevancia a la formación para la enseñanza del LOE y reconocen que esta área resulta estructurante del resto de la formación; 2) No obstante la centralidad de la formación para la enseñanza del LOE, las carreras encuentran restricciones estructurales para profundizar en esta área, fundamentalmente vinculadas a la disponibilidad de recursos y del capital cultural de sus estudiantes; 3) No existe una aproximación homogénea para la formación del LOE, las carreras abordan esta tarea desde una variedad de perspectivas teóricametodológicas que presentan distintos patrones y desbalances en cuanto a la consideración de las dimensiones del conocimiento práctico.

\subsection{RELEVANCIA Y RESTRICCIONES EN LA FORMACIÓN PARA LA ENSEÑANZA DEL LOE}

En las diez carreras analizadas, la formación para la enseñanza del LOE constituye un componente altamente relevante y estructurante, como se observa tanto del análisis estructural de las respectivas mallas y planes de estudio, como en lo declarado por las jefas de carrera.

Ello se evidencia, en primer lugar, en el hecho de que en nueve de los diez planes de estudio analizados esta área está presente a través de cursos que expresamente abordan el LOE (ya sea como línea curricular o como núcleo de especialización), como se observa en el Gráfico 1. Cabe destacar que se presenta una gran diversidad en términos de número (varían entre 1 y 6 cursos dentro del plan común) y de posición en la secuencia curricular, y que no fue posible comparar las horas totales asignadas al LOE en la formación, debido a las diferentes unidades de medida utilizadas para reportar esto en los planes de estudio (y la ausencia de un sistema de créditos transferible en nuestro país).

No obstante las diferencias estructurales ya descritas, la formación del LOE aparece simbólicamente muy protagónica en el discurso. Las diez jefas de carrera dan cuenta de un contexto institucional que releva y a la vez constriñe la enseñanza del LOE como variable crítica en la preparación de las educadoras de párvulos. De esta manera, tres jefas de carrera declararon que la formación para la enseñanza del LOE estructura y articula el resto de la formación en sus instituciones, a través, por ejemplo, de la definición de una base de literatura común sobre LOE para diversos cursos del programa de formación. Más todavía, en aquellas carreras de formación que se encontraban en proceso de renovación o de reforma de sus mallas curriculares, sus jefas de carrera declararon el propósito institucional de fortalecer aún más la formación para la enseñanza del LOE.

De hecho, eso es algo que nosotros estamos trabajando en la malla nueva. En la malla nueva sí hay una orientación clara y está dentro de los contextos de los convenios de desempeño. Lenguaje es una de las áreas que cae como foco de acción en términos de llevar todos los cursos hacia una línea más específica del Pedagogical Content Knowledge y van a haber pasantías. Hay equipos que están trabajando para unificar esos programas, para darles continuidad. Todo bajo una mirada común (Jefa de Carrera 1).

Sin perjuicio de lo anterior, todas las jefas de carrera reconocieron insuficiencias en la formación para la enseñanza del LOE, debido a restricciones estructurales que afectan a las carreras. Entre éstas se encuentran, principalmente, el número de créditos totales que deben integrar el plan de estudios, el perfil de sus estudiantes, las dificultades para integrar la teoría y la práctica en la formación; y las dificultades para que los centros de práctica 
permitan a las estudiantes en práctica realizar experiencias de aprendizaje coherentes con la formación recibida en la carrera.

En cuanto al primer punto, ocho de las diez jefas de carrera declararon que la formación para la enseñanza del LOE se ve constreñida por sus respectivos reglamentos institucionales, los que definen un número máximo de créditos para la carrera. En consecuencia, debido a la orientación generalista de la formación de educadoras de párvulos -la que requiere abordar necesariamente otras áreas del aprendizaje, como las matemáticas, las artes, la comprensión del medio, entre otras- la asignación de créditos para formar a las estudiantes para la enseñanza del LOE no puede ser expandida significativamente, pues implicaría un desmedro de las otras áreas que también son centrales en educación parvularia. De esta manera, según estas jefas de carrera, sus respectivos programas de formación ofrecen actualmente un número de créditos cercano al máximo posible, aunque con la consecuencia de no poder cubrir exhaustivamente todos los contenidos relevantes para la enseñanza del LOE.

Gráfico 1: Número de créditos de cursos sobre LOE respecto del total de créditos del plan de estudios. ${ }^{2}$

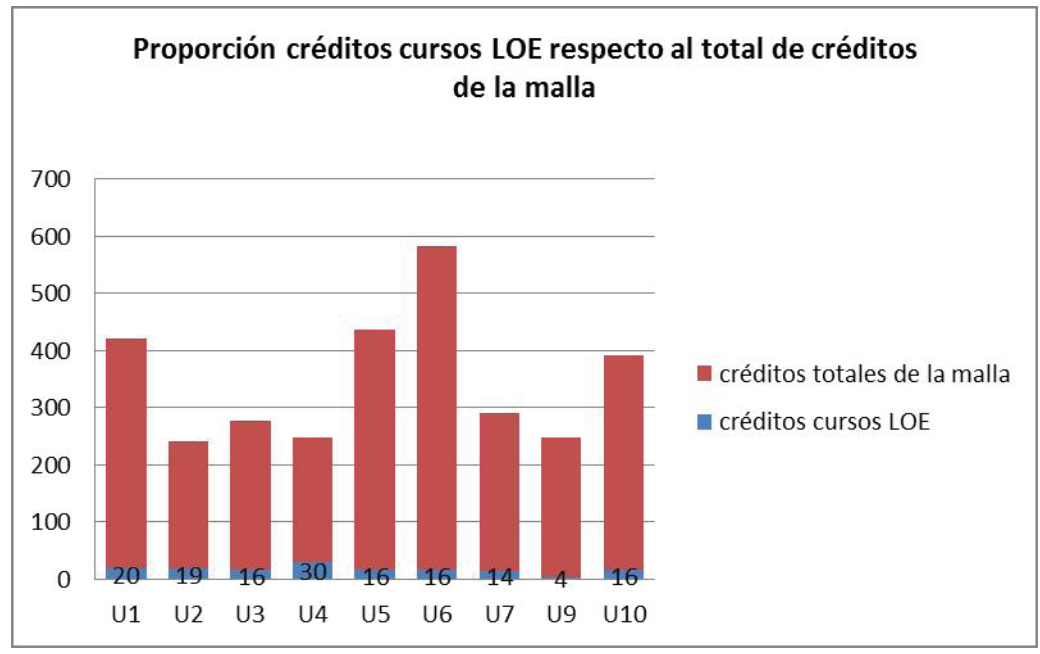

Una segunda limitación deriva del hecho de que cinco de las diez jefas de carrera entrevistadas declararon las débiles competencias lingüísticas y académicas de sus estudiantes como una barrera para profundizar en la formación para la enseñanza del LOE. Concretamente, señalaron que éstas evidencian limitadas capacidades para expresarse oralmente y por escrito en un nivel universitario, y que, además, no han desarrollado ni actitudes, ni hábitos ni técnicas de estudio, así como tampoco habilidades de comprensión

Cabe notar que la Universidad 8 no proporcionó información sobre los créditos que componen el plan de estudios y los cursos. 
de lectura avanzada. Más todavía, una jefa de carrera declaró que los cursos sobre LOE son los que mayor dificultad presentan a las estudiantes debido a estas debilidades. Por tanto, según estas jefas de carrera, sus respectivos programas de formación han debido incluir entre las actividades curriculares regulares algunas de tipo propedéutico, con el fin de fortalecer estas competencias lingüísticas y comunicativas en sus estudiantes. Según su visión, esto implica que la formación para el LOE pierde en profundidad respecto de lo establecido en el programa de los cursos.

Sí, me parece que es insuficiente, porque efectivamente, haciéndonos cargo de las características con las cuales a nosotros nos corresponde realizar una formación profesional de nuestros estudiantes (...) el dato duro que tenemos, es que vienen con todas estas competencias bastante descendidas. Por lo tanto, hacer que nuestros alumnos incorporen todo aquello que no han hecho ni durante la enseñanza básica ni durante la enseñanza media, se nos hace cuesta arriba en estos 4 años de formación (Jefa de Carrera 10).

Complementariamente, seis jefas de carrera señalaron como un desafío de sus respectivos programas superar las dificultades para que las estudiantes integren lo aprendido teóricamente sobre el LOE en sus experiencias de formación práctica profesional. Según explicaron, este problema lo atribuyen a inadecuaciones del modelo formativo, el cual mantendría escindidas estas dos dimensiones. Al respecto, dos jefas de carrera declararon el propósito institucional de enfatizar la formación para la enseñanza del LOE sobre la base del concepto de Conocimiento Pedagógico del Contenido, el cual integra teoría y práctica. Una razón para explicar estas dificultades son las limitaciones que los centros de práctica imponen a las estudiantes, al no permitirles llevar a la práctica lo aprendido en la formación lectiva.

Finalmente, las restricciones de recursos que afectan a tres de las carreras analizadas limitan la posibilidad de conformar un cuerpo docente estable y especializado que asuma un rol de acompañamiento estrecho en la formación teórica y práctica de las estudiantes.

\subsection{PERSPECTIVAS TEÓRICAS DIVERSAS EN LA FORMACIÓN PARA LA ENSEÑANZA DEL LOE}

De acuerdo con lo declarado por las jefas de carrera, es posible identificar tres aproximaciones teóricas para la formación para la enseñanza del LOE en las carreras de formación estudiadas.

Una primera perspectiva es de índole crítica, según la cual la formación para la enseñanza del LOE conlleva un cuestionamiento de los modelos de enseñanza y enfoques de alfabetización centrados en modelos de destrezas predominantes, considerados escolarizantes. Estas carreras buscan la formación de una educadora de párvulos reflexiva, capaz de resignificar la teoría dominante de la enseñanza del LOE, para desarrollar inductivamente prácticas pedagógicas pertinentes y relevantes para los niños.

[...] también en las otras didácticas hay una postura también crítica respecto del marco curricular. No es como: ya, este es el marco curricular y a esto... [...] ven estrategias fundamentalmente que provienen desde modelos holísticos o integrados. $O$ sea, no es que no analicen lo que pasa desde un modelo de destreza; o sea, se parte desde los tres modelos y se van analizando cuáles son las estrategias propias, y ahí aparecen, por ejemplo, la escritura de arabescos, el uso del cuaderno... Pero, fundamentalmente, se cuestionan qué hay detrás, desde 
el punto de vista del aprendizaje significativo, qué significa escribir en ese contexto, cuando te hacen copiar, y fundamentalmente ellas buscan estrategias que desarrollen competencias lectoras y escritoras (Jefa de Carrera 9).

Una segunda perspectiva es de índole disciplinar, en la cual las disciplinas tales como la psicolingüística y las teorías de adquisición y desarrollo de la lengua, son centrales para consolidar en las estudiantes conocimientos avanzados para la enseñanza del LOE. Estas carreras adoptan un enfoque teórico deductivo en la formación pedagógica, según el cual se espera que las estudiantes constaten y apliquen los marcos teóricos revisados en contextos de aula.

Es este foco fuertemente instalado en lo disciplinario. Y eso tiene todo un sentido para nosotros, en el fondo, que tiene que ver con lo que hoy día la literatura dice: que en la medida de que un profesor mientras más competente en términos disciplinarios sea, efectivamente es un profesor que va a disponer de un repertorio mucho más amplio de recursos, de estrategias porque va a poder pensar mucho mejor cómo ese contenido se comporta a la luz de esos recursos y esas estrategias. Entonces, en el fondo, como que estamos un poco dando vuelta el argumento de la educación parvularia que está muchas veces mucho más situada en la perspectiva más de lo metodológico que del contenido propiamente tal. Nosotros estamos bien preocupadas de al revés (Jefa de Carrera 4).

La tercera perspectiva es de índole pragmática, según la cual los planes de estudios se orientan a que las estudiantes conozcan y apliquen un amplio repertorio de estrategias pedagógicas, metodologías o didácticas para la enseñanza del LOE. La orientación de la formación en estas carreras está en el dominio de prácticas efectivas de adquisición de la lengua y la alfabetización, a través de técnicas tales como la lectura compartida, los juegos verbales (rimas y trabalenguas), la sala letrada y las caminatas de lectura, entre otras.

Ellas van trabajando tanto con la profesora de la asignatura, con la asignatura de práctica, en generar una instancia de aprendizaje para los niños que tenga un contenido claro, un buen desarrollo de la actividad, con un buen inicio, un buen desarrollo y un buen cierre de la actividad, con los objetivos claros, los indicadores de evaluación, el material adecuado [...] Nosotros, las profesoras que somos a su vez tutoras de práctica, estamos observando este aprendizaje, estamos haciendo sugerencias y vamos re alimentando y retroalimentando su proceso. ¿Y qué se espera que la alumna aprenda? el cómo debe usar su conocimiento para enseñar a los niños (Jefa de Carrera 7).

\subsection{DESIGUAL ÉNFASIS DE LAS DIMENSIONES DEL CONOCIMIENTO PRÁCTICO EN LA FORMACIÓN PARA LA ENSEÑANZA DEL LOE}

El análisis de los programas de los cursos sobre LOE y de los discursos de las jefas de carrera refleja un desigual énfasis en la dimensión del conocimiento práctico, como se observa en el Gráfico 2. Es decir, algunas dimensiones se encuentran presentes en un alto número de objetivos y unidades de aprendizaje dentro de los cursos sobre LOE. A la vez estas dimensiones son claramente identificables dentro del discurso de las jefas de carrera. Por contraste, otras dimensiones se encuentran escasamente presentes en las carreras de los cursos sobre LOE, siendo además incipientemente descritas en el discurso de las jefas de carrera. 
Gráfico 2: Dimensiones del Conocimiento Práctico presentes en las unidades de aprendizaje/ objetivos de los cursos sobre LOE.

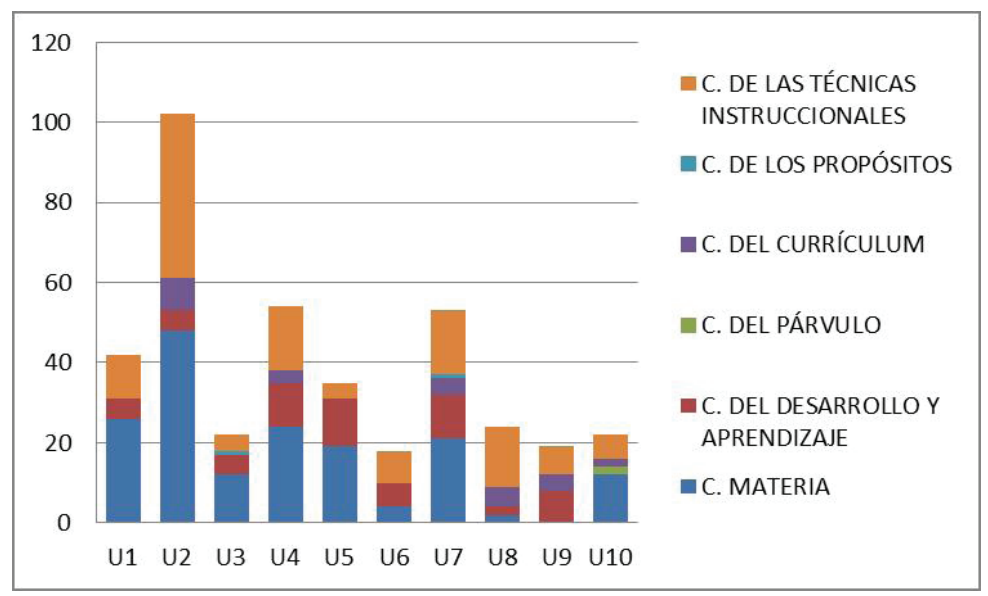

Dentro del primer grupo se encuentran las dimensiones de Conocimiento de Propósitos, Conocimiento de Estrategias Pedagógicas, Conocimiento de Desarrollo y Aprendizaje, y Conocimiento de la Materia. En el segundo grupo, las dimensiones de Conocimiento del Currículum y Conocimiento del Párvulo.

Respecto a la dimensión de Conocimiento de Propósitos, los resultados muestran que, no obstante las diversas perspectivas teóricas adoptadas en las carreras, todas las jefas de carrera señalaron que el propósito que hace relevante la enseñanza del LOE es explícitamente presentado a las estudiantes. De manera consensual, este propósito es la formación al servicio de la integración del niño a la sociedad, al entregarle herramientas para la comunicación o relación con otros, su participación social y el ejercicio de sus derechos. En segundo término, en cinco carreras se complementa lo anterior con propósitos vinculados al goce estético del LOE por parte del niño, a través de obras literarias. Empero, en los programas de los cursos sobre LOE esta dimensión aparece escasamente explicitada.

La dimensión Conocimiento de Estrategias Pedagógicas es la que más se declara en las carreras de los cursos sobre LOE y a la vez la más matizada por las jefas de las carreras analizadas. Todas las carreras analizadas enfatizan que estas estrategias representan una integración de la teoría y la práctica para la enseñanza del LOE, si bien la vinculación entre estos dos ámbitos supone variaciones dependiendo del carácter más crítico, pragmático o disciplinar de cada una. A manera de ejemplo de estrategias pedagógicas para la enseñanza del LOE, se mencionaron el uso de ambientes letrados y de textos auténticos, al interior de las carreras que adoptan una aproximación crítica; la lectura en voz alta y actividades para el desarrollo de la conciencia fonológica, en las carreras que suscriben una perspectiva disciplinar; y el uso del juego y el modelaje, en las carreras de perspectiva pragmática. De todos modos, se aprecian estrategias transversales a todas las carreras analizadas, como la lectura de cuentos y experiencias para el goce de la literatura infantil. Finalmente, cabe destacar que otro foco común en la formación del LOE es el manejo integrado de estrategias de planificación y evaluación de la enseñanza. 
La dimensión del Conocimiento del Desarrollo y Aprendizaje es intencionada en todas las carreras analizadas, exceptuando una sola (donde esta dimensión no se encontró explicitada en la malla curricular). En las carreras que la abordan, un número menor de objetivos o unidades de aprendizaje se enfocan en ella. No obstante, las jefas de carrera le atribuyen gran relevancia, refiriendo a dos grandes vertientes teóricas sobre el desarrollo y el aprendizaje en la primera infancia: una situada en un marco conceptual cognitivista (derivado de la obra de Jean Piaget) y otro de perspectiva sociocultural (proveniente del trabajo de Lev Vigotsky). De todos modos, reconocen una debilidad en la formación respecto a esta dimensión, pues consideran que los cursos son insuficientes o bien poco profundos para abordar los contenidos necesarios.

La dimensión Conocimiento de la Materia también se encuentra presente en una amplia mayoría de las carreras analizadas, con un dispar número de unidades de aprendizaje y objetivos dentro de cada curso sobre LOE. Los contenidos orientados a esta dimensión se concentran en temas de adquisición de la lengua y alfabetización inicial. Con distintos enfoques, en algunas carreras, la formación para el Conocimiento de la Materia se plasma en cursos disciplinares, complementados con cursos didácticos; y en otras, en cursos que integran disciplina y didáctica. Para siete de las diez jefas de carrera entrevistadas, esta es una dimensión relevante, pues entrega el fundamento teórico de la enseñanza del LOE; en las demás, se enfatiza un modelo integral del desarrollo, donde el LOE no cobra relevancia en sí mismo.

En cuanto a la dimensión Conocimiento del Currículum, es interesante observar que se encuentra explícitamente presente en los cursos sobre LOE de sólo tres de las diez carreras analizadas. Sin embargo, nueve jefas de carrera señalaron que las Bases Curriculares de la Educación Parvularia definen los contenidos de los cursos sobre LOE, a partirdelos aprendizajes esperados, ya sea para alinearse a ellas o para abordarlas críticamente (la jefa de la décima carrera declara no estar alineada a ningún referente curricular). Complementariamente, estas jefas de carrera declararon que sus respectivos programas de formación también contemplan otras modalidades curriculares relevantes en educación parvularia, las que ofrecen formatos para la enseñanza del LOE, como por ejemplo, cómo alfabetizar desde la perspectiva Montessori, cómo trabajar proyectos literarios en el currículum Reggio Emilia o bien cómo desarrollar la estrategia de centro de interés del Currículum Integral.

Referente a la dimensión Conocimiento del Párvulo, el análisis de las mallas muestra que éste no se encuentra declarado en ningún componente del plan de estudios de las carreras de formación analizados. Si bien las diez jefas de carrera la reconocen como fundamental en la formación de las educadoras de párvulos, dan cuenta de esta dimensión en términos poco específicos, refiriendo simplemente a la importancia de reconocer atributos de los párvulos importantes de ser considerados en la enseñanza del LOE, como por ejemplo sus intereses, habilidades, contexto familiar y cultural, entre otros. Como métodos para que las estudiantes logren conocer a los niños que tienen a su cargo, destacaron la observación en la formación práctica y el trabajo con las familias de su grupo, entre otros.

\section{CONCLUSIONES Y DISCUSIÓN}

El presente estudio ha proporcionado evidencia sobre la formación para la enseñanza del LOE, a partir del análisis de diez carreras de educación parvularia. Los resultados obtenidos representan una contribución en esta línea de investigación por cuanto expanden 
la escasa evidencia anterior disponible sobre formación en educación parvularia en Chile, al basarse en un marco conceptual que permite interpretar los datos recolectados. Asimismo, este estudio expande el uso del concepto de conocimiento práctico hacia la educación parvularia, aportando a la comprensión del conocimiento que es propio de los educadores en este nivel educativo.

Los resultados obtenidos indican que las diez carreras analizadas estructuran y orientan la formación de sus estudiantes para la enseñanza del LOE en importante concordancia con el estado del arte internacional en esta área. Es decir, la evidencia recogida muestra que en estas carreras se asigna relevancia a la formación para la enseñanza del LOE, abordándola de acuerdo con debates vigentes, y considerando temas señalados como importantes en la literatura. Como característica singular, se debe observar la diversidad de enfoques presentes en estas carreras (i.e. disciplinar, crítico y pragmático), derivados de un marco institucional más amplio.

De particular interés resulta notar que el ejercicio de analizar la formación para la enseñanza del LOE en torno a las dimensiones del conocimiento práctico muestra un importante desbalance entre estas dimensiones. Si bien este concepto no forma parte de los enfoques teóricos de las diez carreras analizadas, sí es concordante con la visión holística de la práctica pedagógica que representa un precepto en la educación parvularia. Por tanto, el hallazgo de que algunas dimensiones del conocimiento práctico son intencionadas de forma incipiente puede indicar limitaciones en estos aspectos de la formación de educadoras de párvulos en esta área, como lo reconocen las diez jefas de carrera. Por otra parte, resulta interesante observar que dos de las carreras analizadas están comenzando a incorporar conceptos equivalentes al de conocimiento práctico (i.e. pedagogical content knowledge), sugiriendo que la relevancia de esta noción está ganando reconocimiento en la formación de educadoras de párvulos en el país.

Finalmente, los hallazgos obtenidos muestran que las limitaciones de la formación de educadoras de párvulos para la enseñanza del LOE son compartidas en gran medida en las diez carreras analizadas, pese a los diferentes enfoques a que adhiere cada una. Algunas de estas limitaciones son derivaciones de la estructura de nuestro sistema de educación superior, como las características de las estudiantes y las limitaciones de recursos al interior de las carreras. Otras, en cambio, derivan de disyuntivas que internacionalmente enfrenta la educación de educadoras de párvulos para la enseñanza del LOE, como el tiempo asignado a esta área dentro de un marco de formación generalista y las complejidades de la formación práctica. Por tanto, estos resultados sugieren que, con miras a fortalecer la formación para la enseñanza del LOE, las carreras analizadas deben enfrentar dilemas estructurales formidables, cuya solución no es evidente ni sencilla.

\section{REFERENCIAS BIBLIOGRÁFICAS}

Adger, C., Snow, C., \& Christian, D. (2003). What teachers need to know about language. Washington, DC: Center for Applied Linguistics.

Bank Street College. (2013). M.S. Ed. Degree Early Childhood and Childhood General Education. Recuperado el 28-04-2014, desde: http://bankstreet.edu/graduate-school/academics/programs/ general-teacher-education-programs-overview/early-childhood-and-childhood-generaleducation/ 
Barnett, S. (2003). Better teachers, better preschools: student achievement linked to teacher qualifications. Preschool Policy Matters, 2. New York: National Institute for Early Education Research (NIEER).

Beijaard, D., Meijer, P. \& Verloop, N. (2004). Reconsidering research on teachers' professional identity. Teaching and Teacher Education, vol.20, n.2, 107-128.

Beijaard, D., Verloop, N. (1996). Assessing teachers' practical knowledge. Studies In Educational Evaluation, vol.22, n.3, 275-286.

Beijaard, D. (1995). Teachers' prior experiences and actual perceptions of professional identity. Teachers and Teaching: Theory and Practice, vol.1, n.2, 281-294.

Boston College. (2013). M.Ed./ Early Childhood Education. Recuperado el 28-04-2014, desde: http://www.bc.edu/content/dam/files/schools/lsoe/pdf/pos/Fall2013/2013PDFS/2013TE_MEd_ Early Child-7-10-13.pdf

Bowman, B., Donovan, M. \& Burns, S. (2000). Eager to Learn: Educating Our Preschoolers. Washington, DC: The National Academies Press.

Bredekamp, S. (2013). Making the case: Why credentials and certification matter. En: R. Pianta, S. Barnett, L. Justice \& S. Sheridan (Ed.), Handbook of early childhood education (pp. 584-604). New York, NY: The Guilford Press.

(2003). Language and early childhood programs. En: C. Adger, C. Snow, \& D. Christian (Eds.), What teachers need to know about language (pp. 55-69). Washington, DC: National Library of Education - Carnegie Corporation.

Brown, S. \& McIntyre, D. (1992). Making sense of teaching. London: Routledge.

Burchinal, M., Cryer, D. \& Clifford, R. (2002). Caregiver training and classroom quality in child care centers. Applied Developmental Science, vol.6, n.1, 2-11.

Burney, D. (2004). Craft knowledge: The road to transforming schools. Phi Delta Kappan, vol.85, n.7, 526-531.

Burns, M., Griffin, P. \& Snow, C. (1999). Starting Out Right: A Guide to Promoting Children's Reading Success. Washington, DC: The National Academies Press.

Calderhead, J. (1991). The nature and growth of knowledge in student teaching. Teaching and Teacher Education, vol.7, n.6, 531-535.

Clandinin, D. J. \& Connelly, F. M. (1988). Studying teachers' knowledge of classrooms: Collaborative research, ethics and the negotiation of narrative. The Journal of Educational Thought, vol.22, n.2A, 269-282.

Cochran, K. F., De Ruiter, J. A. \& King, R. A. (1993). Pedagogical content knowing: An integrative model for teacher preparation. Journal of Teacher Education, vol.44, n.4, 263-272.

Cochran-Smith, M. (2010). The future of teacher education: Framing the questions that matter. Teaching Education, vol.11, n.1, 13-24.

Connelly, F. M., Clandinin, D. J. \& He, M. F. (1997). Teachers' personal practical knowledge on the professional knowledge landscape. Teaching and Teacher Education, vol.13, n.7, 665-674.

Cusumano, D. L., Armstrong, K., Cohen, R. \& Todd, M. (2006). Indirect impact: How early childhood education training and coaching impacted the acquisition of literacy skills in preschool students. Journal of Early Childhood Teacher Education, vol.27, n.4, 363-377.

Domínguez, M., Bascopé, M., Meckes, L. y San Martín, E. (2012). ¿Producen mejores resultados las carreras de pedagogía básica con más años de acreditación? Estudios Públicos, n.128, 1-59.

Early, D. M. \& Winton, P. J. (2001). Preparing the workforce: early childhood teacher preparation at 2- and 4-year institutions of higher education. Early Childhood Research Quarterly, vol.16, n.3, 285-306.

Elbaz, F. (1983). Teacher thinking: A study of practical knowledge. London: Croom Helm.

Feldman, S. (2003). Preparing teachers to guide children's language development. En C. Adger, C. Snow, \& D. Christian (Ed.), What teachers need to know about language (pp. 113-122). Washington, DC: Center for Applied Linguistics. 
Estudios Pedagógicos XLII, No 1: 17-36, 2016

FORMACIÓN PARA LA ENSEÑANZA DEL LENGUAJE ORAL Y ESCRITO EN CARRERAS DE EDUCACIÓN

PARVULARIA: VARIEDAD DE APROXIMACIONES Y SIMILARES DILEMAS

Fernández-Balboa, J. M. \& Stiehl, J. (1995). The generic nature of pedagogical content knowledge among college professors. Teaching and Teacher Education, vol.11, n.3, 293-306.

Fillmore, L. \& Snow, C. (2000). What teachers need to know about language. En C. Adger, C. Snow, \& D. Christian (Ed.), What teachers need to know about language (pp. 7-53). Washington, DC: Center for Applied Linguistics.

Freidson, E. (1986). Professional powers. A study of the institutionalization of formal knowledge. Chicago, IL: The University of Chicago Press.

Garcia-Huidobro, J. (2006). Formación inicial de educadoras (es) de párvulos en Chile. Santiago, Chile: Seminario Expansiva.

Genishi, C., Ryan, S., Ochsner, M. \& Yarnall, M. (2001). Teaching in early childhood education: Understanding practice through research and theory. En: V. Richardson (Ed.), Handbook of research on teaching (pp. 1175-1210). Washington, DC: American Educational Research Association.

Grossman, P. (2005). Research on pedagogical approaches in teacher education. En: M. CochranSmith \& K. Zeichner (Ed.), The report of the AERA panel on research and teacher education. Mahweh, N.J.: Lawrence Erlbaum Publishers.

. (2007). Fundamentos de metodología de la investigación. México, D.F.: McGraw-Hill.

(1990). The making of a teacher: Teacher knowledge and teacher education. New York: Teachers College Press.

Hernández Sampieri, R., Fernández, C., y Baptista, M. (2010). Metodología de la investigación. Perú: McGraw-Hill.

. (2007). Fundamentos de metodología de la investigación. México, D.F.: McGraw-Hill.

Howey, K. \& Grossman, P. (1989). A study in contrast: Sources of pedagogical content knowledge for secondary English. Journal of Teacher Education, vol.40, n.5, 24-31.

Hyson, M., Horn, D. \& Winton, P. (2013). Higher education for early childhood educators and outcomes. En: R. Pianta, S. Barnett, L. Justice \& S. Sheridan (Ed.), Handbook of early childhood education (pp. 553-583). New York, NY: The Guilford Press.

Institute of Education University of London. (2013). BA / BEd Education (Honours Top-up Programme) - Working With Children: Education And Well-Being Route. Recuperado el 10-032014, desde: http://www.ioe.ac.uk/study/IBES_EDU99P.html

International Reading Association - National Association for the Education of Young Children. (1998). Learning to read and write: Developmentally appropriate practices for young children. Washington, DC: NAEYC.

Krieg, S. (2010). The professional knowledge that counts in Australian contemporary early childhood teacher education. Contemporary Issues in Early Childhood, n.11, 144-155.

Lenz Taguchi, H. (2007). Deconstructing and transgressing the theory-practice dichotomy in early childhood education. Educational Philosophy and Theory, vol.39, n.3, 275-290.

Maakestad Wolf, J. (1999). Making connections: From the college classroom to the early childhood setting. Journal of Early Childhood Teacher EducationEducation, vol.20, n.2, 167-172.

Manzi, J. (2011). ¿Qué características de la formación inicial de los docentes se asocian a mayores avances en su aprendizaje de conocimientos disciplinarios? Santiago, Chile: Ministerio de Educación.

Marks, R. (1990). Pedagogical content knowledge: From a mathematical case to a modified conception. Journal of Teacher Education, vol.41, n.3, 3-11.

McLean, V. (1992). Developing personal practical knowledge in early childhood teacher education. Paper presented at the World Congress of the World Organization for Early Childhood Education.

McNamara, D. \& Desforges, C. (1978). The social sciences, teacher education and the objectification of craft knowledge. British Journal of Teacher Education, vol.4, n.1, 17-36.

Meijer, P., Verloop, N. \& Beijaard, D. (2002). Multi-Method Triangulation in a Qualitative Study on Teachers' Practical Knowledge: An Attempt to Increase Internal Validity. Quality y Quantity, 
n.36, 145-167.

. (2001). Similarities and Differencies in Teachers' Practical Knowledge about Teaching Comprehension. The Journal of Educational Research, vol.94, n.3, 171-184.

(1999). Exploring language teachers' practical knowledge about teaching reading comprehension. Teaching and Teacher Education, n.15, 59-84.

Mills College. (2014). Education. Recuperado el 29-04-2014, desde: http://www.mills.edu/ academics/undergraduate/educ/degree_requirements.php\#4+1CDev

Ministerio de Educación. (2014). Base de datos oferta académica. Recuperado el 30-03-2014, desde: http://www.mifuturo.cl/

Mischo, C., Wahl, S., Strohmer, J. \& Wolf, C. (2014). Does early childhood teacher education affect students' cognitive orientations? Journal of Education and Training Studies, vol.2, n.1, 193-206.

Monash University. (2013). Bachelor of Early Childhood Education for 2013. Recuperado el 10-032014, desde: http://www.monash.edu.au/study/coursefinder/course/1515/

Munby, H., Russell, T. \& Martin, A. (2001). Teachers' knowledge and how it develops. En V. Richardson (Ed.), Handbook of research on teaching (pp. 877-904). Washington, DC: American Educational Research Association.

National Institute of Child Health and Human Development. (2000). Report of the National Reading Panel. Teaching children to read: An evidence-based assessment of the scientific research literature on reading and its implications for reading instruction. Washington, DC: National Institute of Health.

National Institute of Education. (2013). Master of Education. Singapore: Office of Graduate Studies and Professional Learning.

Neuman, S. (2013). Improving language and literacy outcomes in child care. En R. Pianta, S. Barnett, L. Justice \& S. Sheridan (Ed.), Handbook of early childhood education (pp. 533-554). New York, NY: The Guilford Press.

Neuman, S. \& Kamil, M. (2010). Preparing Teachers for the Early Childhood Classroom. Proven Models y Key Principles. Baltimore, MD: Paul H. Brooks Publishing.

Panel de Expertos para una Educación de Calidad. (2010). Propuestas para fortalecer la profesión docente en el sistema escolar chileno. Santiago, Chile: Gobierno de Chile.

Ponte, P., Ax, J., Beijaard, D. \& Wubbels, T. (2004). Teachers' development of professional knowledge through action research and the facilitation of this by teacher educators. Teaching and Teacher Education, n.20, 571-588.

Ray, A., Bowman, B. \& Robbins, J. (2006). Preparing early childhood teachers to successfully educate all children: The contribution of state boards of higher education and national professional accreditation organizations. Chicago, Il: Foundation for Child Development.

Rojas, M., Gorichon, S., Falabella, A. y Lee, M. (2008). Propuestas de mejoramiento para la formación de profesionales de educación parvularia. Santiago, Chile: Universidad Alberto Hurtado.

Romo, V., Simonstein, S., Peralta, V. y Mayorga, L. (2009). Especialización en la formación inicial y continua de educadores de párvulos en los primeros cuatro años de vida de niños/as. Santiago, Chile: Universidad Central.

Sahakian, C. (1997). The Delphi Method. Chicago, IL: Corporate Partnering Inst.

Saracho, O. \& Spodek, B. (2001). Contemporary Perspectives on Early Childhood Curriculum. Greenwich, CT: Information Age Publishing.

Shonkoff, J. \& Phillips, D. (Eds.). (2000). From neurons to neighborhood. The science of early childhood development. Washington, DC: National Academy Press.

Shulman, L. \& Shulman, J. (2004). How and what teachers learn: a shifting perspective. Journal of Curriculum Studies, vol.36, n.2, 257-271.

Shulman, L. (1987). Foundations of the new reform. Harvard Educational Review, n.57, 1-22.

Sotomayor, C., Coloma, C. J., Parodi, G., Ibáñez, R., Cavada, P. y Gysling, J. (2013). Percepción de los estudiantes de pedagogía sobre su formación inicial. Magis, Revista Internacional de 
Investigación En Educación, vol.5, n.11, 375-392.

Spodek, B. \& Saracho, O. (1990). Preparing early childhood teachers. En B. Spodek \& O. Saracho (Ed.), Early Childhood Teacher Preparation. New York, NY: Teachers College Press.

Strauss, A. y Corbin, J. (2002). Bases de la investigación cualitativa. Técnicas y procedimientos para desarrollar la teoría fundamentada. Medellín: Editorial Universidad de Antioquía.

Strickland, D., Snow, C., Griffin, P., Burns, S. \& McNamara, P. (2002). Preparing our teachers: Opportunities for better reading instruction. Washington, DC: John Henry Press.

Tashakkori, A. \& Teddle, C. (2003). Handbook of Mixed Methods in Social and Behavioral Research. Thousand Oaks: SAGE Publications.

Teachers College, C. U. (2014). MA: Early Childhood Education-Initial Certification. Recuperado el 28-04-2014, desde: http://www.tc.columbia.edu/cyt/childed/index.asp?Id=DegreesyInfo=MA: + Early+Childhood+Education-Initial+Certification

Universidad de Ciencias Pedagógicas Enrique José Varona. (2013). Carrera Licenciado en Educación. Especialidad en Educación Preescolar. La Habana, Cuba.

University of Cambridge Faculty of Education. (2014). Postgraduate Certificate of Education. Prospectus 2014-2015. Recuperado el 10-06-2013, desde: http://www.educ.cam.ac.uk/courses/ pgce/downloads/2014_Prospectus.pdf

University of Oulu. (2013). Early Childhood Education. Bachelor of Arts. Recuperado el 10-032014, desde: http://www.oulu.fi/edu/early_childhood_education

University of Toronto. (2013). Master of Arts in Child Study and Education. Recuperado el 30-082014, desde: http://www.oise.utoronto.ca/ics/M.A._Program/index.html

Valdés, G., Bunch, G., Snow, C., Lee, C. \& Matos, L. (2005). Enhancing the Development of Students' Language(s). En L. Darling-Hammond \& J. Bransford (Ed.), Preparing teachers for a changing world: what teachers should learn and be able to do. San Francisco, CA: National Academy of Education.(126-168).

Van Driel, J., Beijaard, D. \& Verloop, N. (2001). Professional Development and Reform in Science Education: the Role of Teachers' Practical Knowledge. Of Research in Science Teaching, vol.38, n.2, 137-158.

Viernickel, S. y Tietze, W. (2010). Desarrollo de la Calidad Educativa en Centros Preescolares. Catálogo de Criterios de Calidad. Santiago: LOM Ediciones.

Wheelock College. (2013). Early Childhood Education. Recuperado el 10-03-2014, desde: http:// www.wheelock.edu/academics/undergraduate/majors-minors-certificates/early-childhoodeducation\#course

Whitebook, M., Austin, L., Ryan, S., Kipnis, F., Almaraz, M. \& Sakai, L. (2012). By default or by design? Berkeley, CA: Center for the Study of Child Care Employment.

Whitebook, M., Gomby, D., Bellm, D., Sakai, L. \& Kipnis, F. (2009). Preparing Teachers of Young Children: The Current State of Knowledge, and a Blueprint for the Future. Berkeley, CA: Center for the Study of Child Care Employment.

Whitebook, M., Bellm, D., Lee, Y. \& Sakai, L. (2005). Time to Revamp and Expand: Early Childhood Teacher Preparation Programs in California's Institutions of Higher Education. Berkeley, CA: Center for the Study of Child Care Employment. 